\title{
Error Rate Analysis of Cognitive Radio Transmissions with Imperfect Channel Sensing
}

\author{
Gozde Ozcan and M. Cenk Gursoy \\ Department of Electrical Engineering and Computer Science \\ Syracuse University, Syracuse, NY 13244 \\ Email: gozcan@syr.edu, mcgursoy@syr.edu
}

\author{
Sinan Gezici \\ Department of Electrical and Electronics Engineering \\ Bilkent University, Bilkent, Ankara 06800, Turkey \\ Email: gezici@ee.bilkent.edu.tr
}

\begin{abstract}
In this paper, error rate performance of cognitive radio transmissions is studied in the presence of imperfect channel sensing decisions. It is assumed that cognitive users first perform channel sensing, albeit with possible errors. Then, depending on the sensing decisions, they select the transmission energy level and employ $M_{I} \times M_{Q}$ rectangular quadrature amplitude modulation (QAM) for data transmission over a fading channel. In this setting, the optimal decision rule is formulated under the assumptions that the receiver is equipped with the sensing decision and perfect knowledge of the channel fading. It is shown that the thresholds for optimal detection at the receiver are the midpoints between the signals under any sensing decision. Subsequently, minimum average error probability expressions for $M-$ ary pulse amplitude modulation $(M-\mathbf{P A M})$ and $M_{I} \times M_{Q}$ rectangular QAM transmissions attained with the optimal detector are derived. The effects of imperfect channel sensing decisions on the average symbol error probability are analyzed.
\end{abstract}

Index Terms-Cognitive radio, PAM, QAM, symbol error probability, fading channel, Gaussian mixture noise, channel sensing.

\section{INTRODUCTION}

Rapid growth in the use of wireless services coupled with inefficient utilization of scarce spectrum resources has led to much interest in the analysis and development of cognitive radio systems. One of the important considerations in cognitive radio systems is to control the interference inflicted on the primary users. In order to limit such interference, cognitive secondary users generally sense the channel for primary user activity before initiating their own transmissions. In the literature, different spectrum sensing methods and dynamic spectrum access strategies have been extensively studied in recent times (see e.g., [1]-[3]). It is important to note that, as common to all schemes, channel sensing is generally performed with errors and such errors can lead to degradation in the performance.

In addition to channel sensing methods, performance analysis of cognitive radio transmissions is also conducted in numerous studies. However, in most works, transmission rate is considered as the main performance metric. For instance, channel capacity under average and peak received-power constraints is studied in [4]. In [5], sensing-throughput tradeoff is investigated in cognitive radio networks.

Recently, the authors in [6] have obtained closed-form bit error rate expression by considering the interference limit of the primary receiver is very high. Also, the work in [7] focuses on the optimal power allocation that minimizes average bit error rate subject to peak/average transmit power and peak/average interference power constraints while the interference on the secondary users caused by primary users is ignored. Moreover, in [8], the opportunistic scheduling in multiuser underlay cognitive radio systems is studied in terms of link reliability. However, in the error rate analysis of above works, channel sensing errors are not taken into consideration.

In this paper, we study the error performance of cognitive radio transmissions when the cognitive users have only imperfect channel sensing decisions. Channel sensing performance is assessed through detection and false-alarm probabilities. In the presence of sensing errors, we note that the secondary users experience Gaussian mixture noise when the primary users' received signal is modeled as Gaussian distributed together with the background noise. In our analysis, we assume that rectangular QAM signaling is employed by the cognitive users for data transmission. We show that the optimal detector sets the threshold midway between the signal points, and then we determine the error probability expressions in closed-form. Through this analysis, we investigate the impacts of imperfect channel sensing on the error-rate performance of cognitive transmissions when the secondary users are assumed to either coexist with the primary users (while lowering their transmission power when the channel is detected as busy) or transmit only when the primary users are not detected in the channel.

\section{SyStem Model}

\section{A. Channel Sensing}

We consider a cognitive radio system in which the secondary users initially sense the channel. Channel sensing can be modeled as a hypothesis testing problem. Assume that $\mathcal{H}_{0}$ denotes the hypothesis that the primary users are inactive in the channel, and $\mathcal{H}_{1}$ denotes the hypothesis that the primary users are active. Various channel sensing methods, including energy detection, cyclostationary detection, and matched filtering, have been proposed and analyzed in the literature. Regardless of which method is used, one common feature is that errors in the form of miss-detections and false-alarms occur in channel sensing. The ensuing analysis takes such errors into account and depends on the sensing scheme only through the detection and false-alarm probabilities. Assume that $\hat{\mathcal{H}}_{0}$ and $\hat{\mathcal{H}}_{1}$ denote the sensing decisions that the primary users are inactive and active, respectively. Then, the detection and false-alarm probabilities can be expressed respectively as the following conditional 
probabilities:

$$
P_{d}=\operatorname{Pr}\left\{\hat{\mathcal{H}}_{1} \mid \mathcal{H}_{1}\right\}, \quad P_{f}=\operatorname{Pr}\left\{\hat{\mathcal{H}}_{1} \mid \mathcal{H}_{0}\right\}
$$

\section{B. Cognitive Channel Model}

Following channel sensing, the secondary transmitter performs data transmission over a flat-fading channel. We assume that the average transmission energy is selected depending on the channel sensing decision. More specifically, the average energy is $\mathcal{E}_{1}$ if primary user activity is detected in the channel (denoted by the event $\hat{\mathcal{H}}_{1}$ ) whereas the average energy is $\mathcal{E}_{0}$ if no primary user transmissions are sensed (denoted by the event $\hat{\mathcal{H}}_{0}$ ). We in general have $\mathcal{E}_{1} \leq \mathcal{E}_{0}$ in order to limit the interference on the primary users. If no transmission is allowed when the channel is detected as busy, then we set $\mathcal{E}_{1}=0$.

Note that as a result of channel sensing decisions and the true nature of primary user activity, we have four possible cases which are described below together with corresponding inputoutput relationships:

- Case (I): A busy channel is sensed as busy $\left(\mathcal{H}_{1}, \hat{\mathcal{H}}_{1}\right)$.

(Correct detection) $\quad y=h s+n+w$

- Case (II): A busy channel is sensed as idle $\left(\mathcal{H}_{1}, \hat{\mathcal{H}}_{0}\right)$.

(Miss-detection) $\quad y=h s+n+w$.

- Case (III): An idle channel is sensed as busy $\left(\mathcal{H}_{0}, \hat{\mathcal{H}}_{1}\right)$.

(False alarm) $\quad y=h s+n$.

- Case (IV): An idle channel is sensed as idle $\left(\mathcal{H}_{0}, \hat{\mathcal{H}}_{0}\right)$.

(Correct detection) $\quad y=h s+n$.

In the above expressions, $s$ is the transmitted signal, $y$ is the received symbol, $h$ denotes zero-mean, circularly-symmetric complex fading coefficient between the secondary transmitter and receiver with variance $\sigma_{h}^{2}$, and $n$ denotes the circularlysymmetric complex Gaussian noise with mean zero and variance $\mathbb{E}\left\{|n|^{2}\right\}=\sigma_{n}^{2}\left(\sigma_{n}^{2} / 2\right.$ per real and imaginary components $)$, i.e., $n \sim \mathcal{C N}\left(0, \sigma_{n}^{2}\right)$. The active primary users' received sum signal at the secondary receiver is denoted by $w$. Notice that if the primary users are active and hence the hypothesis $\mathcal{H}_{1}$ is true as in cases (I) and (II), the secondary receiver experiences interference from the primary users' transmissions in the form of $w$ which we also model as circularly-symmetric complex Gaussian random variable with zero mean and variance $\mathbb{E}\left\{|w|^{2}\right\}=\sigma_{w}^{2}$, i.e., $w \sim \mathcal{C} \mathcal{N}\left(0, \sigma_{w}^{2}\right)$. Hence, not knowing the true state of the primary user activity perfectly, the cognitive secondary receiver effectively experiences Gaussian mixture noise.

\section{Performance Analysis}

In this section, we investigate the optimal decision rule for the cognitive radio system in the presence of channel sensing errors, and conduct an error probability analysis. In subsections III-A and III-B, we provide general formulations applicable to any modulation scheme. More specific analysis on QAM is conducted in subsection III-C.

\section{A. The Optimal Decision Rule}

Remark 1: In the cognitive radio setting considered in this paper, the optimal maximum a posteriori probability (MAP) decision rule under sensing decision $\hat{\mathcal{H}}_{k}$ can be formulated for any arbitrary $M$-ary digital modulation as follows:

$$
\begin{aligned}
\hat{s}=\underset{1 \leq m \leq M}{\arg \max } \operatorname{Pr}\left\{s_{m} \mid y, h, \hat{\mathcal{H}}_{k}\right\} \\
=\underset{1 \leq m \leq M}{\arg \max } P_{m}\left(\operatorname{Pr}\left\{\mathcal{H}_{0} \mid \hat{\mathcal{H}}_{k}\right\} f\left(y \mid s_{m}, h, \hat{\mathcal{H}}_{k}, \mathcal{H}_{0}\right)\right. \\
\left.\quad+\operatorname{Pr}\left\{\mathcal{H}_{1} \mid \hat{\mathcal{H}}_{k}\right\} f\left(y \mid s_{m}, h, \hat{\mathcal{H}}_{k}, \mathcal{H}_{1}\right)\right)
\end{aligned}
$$

where $P_{m}$ is the prior probability of signal $s_{m}$ and $k \in\{0,1\}$. Above, $f\left(y \mid s, h, \hat{\mathcal{H}}_{k}, \mathcal{H}_{j}\right)$ is the conditional distribution of the received real signal $y$ given the transmitted signal $s_{m}$, channel fading coefficient $h$, channel sensing decision $\hat{\mathcal{H}}_{k}$ and true state of the channel $\mathcal{H}_{j}$, can be written as

$$
f\left(y \mid s_{m}, h, \hat{\mathcal{H}}_{k}, \mathcal{H}_{j}\right)= \begin{cases}\frac{1}{\pi \sigma_{n}^{2}} \mathrm{e}^{-\frac{\left|y-s_{m} h\right|^{2}}{\sigma_{n}^{2}}}, & j=0 \\ \frac{1}{\pi\left(\sigma_{n}^{2}+\sigma_{w}^{2}\right)} \mathrm{e}^{-\frac{\left|y-s_{m} h\right|^{2}}{\sigma_{n}^{2}+\sigma_{w}^{2}}}, & j=1\end{cases}
$$

Moreover, conditional probabilities in (7) can be expressed as

$$
\operatorname{Pr}\left\{\mathcal{H}_{j} \mid \hat{\mathcal{H}}_{k}\right\}=\frac{\operatorname{Pr}\left\{\mathcal{H}_{j}\right\} \operatorname{Pr}\left\{\hat{\mathcal{H}}_{k} \mid \mathcal{H}_{j}\right\}}{\sum_{i=0}^{1} \operatorname{Pr}\left\{\mathcal{H}_{i}\right\} \operatorname{Pr}\left\{\hat{\mathcal{H}}_{k} \mid \mathcal{H}_{i}\right\}} \quad j, k \in\{0,1\}
$$

where $\operatorname{Pr}\left\{\mathcal{H}_{0}\right\}$ and $\operatorname{Pr}\left\{\mathcal{H}_{1}\right\}$ are the prior probabilities of the channel being idle and busy, respectively, and the conditional probabilities in the form $\operatorname{Pr}\left\{\hat{\mathcal{H}}_{j} \mid \mathcal{H}_{i}\right\}$ depend on the channel sensing performance. As discussed in Section II-A, $P_{d}=$ $\operatorname{Pr}\left\{\hat{\mathcal{H}}_{1} \mid \mathcal{H}_{1}\right\}$ is the detection probability and $P_{f}=\operatorname{Pr}\left\{\hat{\mathcal{H}}_{1} \mid \mathcal{H}_{0}\right\}$ is the false alarm probability.

From (7), we see that the cognitive secondary receiver under sensing errors detects the received signal $y$ in the presence of symmetric Gaussian mixture noise with zero mean since the received signal $y$ is corrupted by zero mean complex background Gaussian noise $n$ and the sum of primary users' faded signal $w$, which is assumed to be a zero mean complex Gaussian random variable as well.

\section{B. Average Symbol Error Probability}

If the cognitive users are allowed to perform data transmission when primary user activity is detected in the channel, the average symbol error probability for the MAP decision rule in (6) is computed as

$$
\begin{aligned}
& P_{e}=1-\sum_{m=1}^{M} P_{m} \operatorname{Pr}\left\{\hat{s}=s_{m} \mid s_{m}\right\} \\
& =1-\sum_{m=1}^{M} \sum_{k=0}^{1} \sum_{i=0}^{1} P_{m} \operatorname{Pr}\left\{\hat{\mathcal{H}}_{k}\right\}\left(\operatorname{Pr}\left\{\mathcal{H}_{i} \mid \hat{\mathcal{H}}_{k}\right\} \operatorname{Pr}\left\{\hat{s}=s_{m} \mid s_{m}, \mathcal{H}_{i}, \hat{\mathcal{H}}_{k}\right\}\right) .
\end{aligned}
$$

If cognitive user transmission is not allowed in the case of the channel being sensed as occupied by the primary users, the average probability of error can be simply expressed as

$P_{e}=1-\sum_{m=1}^{M} \sum_{i=0}^{1} P_{m}\left(\operatorname{Pr}\left\{\mathcal{H}_{i} \mid \hat{\mathcal{H}}_{0}\right\} \operatorname{Pr}\left\{\hat{s}=s_{m} \mid s_{m}, \mathcal{H}_{i}, \hat{\mathcal{H}}_{0}\right\}\right)$ 
C. Optimal Decision Rule and Average Symbol Error Probability for QAM Modulation

In this section, we conduct a more detailed analysis by considering rectangular QAM to demonstrate the key tradeoffs in a lucid setting. We find the optimal decision regions of equiprobable $M_{I} \times M_{Q}$ rectangular QAM transmissions with sensing errors and identify the error rates. We initially address $M$-PAM modulation. Extension to $M_{I} \times M_{Q}$ rectangular QAM is straightforward as it can be regarded as two non-interacting PAM modulations on the in-phase and quadrature components, i.e., $M_{I}-\mathrm{PAM}$ and $M_{Q}-\mathrm{PAM}$.

1) $M-P A M$ transmission under channel sensing error: The amplitude level of $M$-PAM signal is determined as follows

$$
s_{m}=(2 m-1-M) \frac{d_{m i n, k}}{2}
$$

for $m=1, \ldots, M$ and the minimum $d_{m i n, k}$ distance between the signal points is given by

$$
d_{\min , k}=\sqrt{\frac{12}{M^{2}-1} \mathcal{E}_{k}} \quad k \in\{0,1\}
$$

where $\mathcal{E}_{k}$ is the average energy under sensing decision $\hat{\mathcal{H}}_{k}$.

It is assumed that the fading realizations are perfectly known at the receiver. In this case, phase rotations caused by the fading can be offset by multiplying the channel output $y$ with $\mathrm{e}^{-j \theta_{h}}$ where $\theta_{h}$ is the phase of the fading coefficient $h$. Hence, the modified received signal can be written in terms of its real and imaginary parts as follows:

$$
\begin{aligned}
\bar{y} & =\bar{y}_{r}+j \bar{y}_{i}=y \mathrm{e}^{-j \theta_{h}} \\
& = \begin{cases}s_{r}|h|+\bar{n}_{r}+j\left(s_{i}|h|+\bar{n}_{i}\right) & \text { under } \mathcal{H}_{0} \\
s_{r}|h|+\bar{n}_{r}+\bar{w}_{r}+j\left(s_{i}|h|+\bar{n}_{i}+\bar{w}_{i}\right) & \text { under } \mathcal{H}_{1}\end{cases}
\end{aligned}
$$

where the subscripts $r$ and $i$ are used to denote the real and imaginary components of the signal, respectively. Note that $\bar{n}=$ $\bar{n}_{r}+j \bar{n}_{i}$ and $\bar{w}=\bar{w}_{r}+\bar{w}_{i}$ have the same statistics as $n$ and $w$, respectively, due to their property of being circularly symmetric. Moreover, the real and imaginary parts of noise, i.e., $\bar{n}_{r}$ and $\bar{n}_{i}$, and the real and imaginary parts of primary users' faded sum signal, i.e., $\bar{w}_{r}$ and $\bar{w}_{i}$, are independent zero-mean Gaussian random variables. We can further express the received $M-\mathrm{PAM}$ signal as

$$
\bar{y}= \begin{cases}s|h|+\bar{n}_{r}+j \bar{n}_{i} & \text { under } \mathcal{H}_{0} \\ s|h|+\bar{n}_{r}+\bar{w}_{r}+j\left(\bar{n}_{i}+\bar{w}_{i}\right) & \text { under } \mathcal{H}_{1}\end{cases}
$$

Proposition 1: For cognitive radio transmissions with equiprobable $M$-PAM under channel sensing errors, the optimal detection thresholds for any channel sensing decision are located midway between the received signal points. Hence, the optimal detector does not depend on the sensing decision.

Under the optimal decision rule, the average symbol error probability of the equiprobable signals is given by

$$
P_{e, h}=\sum_{i, k=0}^{1} \operatorname{Pr}\left\{\hat{\mathcal{H}}_{k}\right\}\left(\operatorname{Pr}\left\{\mathcal{H}_{i} \mid \hat{\mathcal{H}}_{k}\right\} \frac{1}{M} \sum_{m=1}^{M} \operatorname{Pr}\left\{e \mid s_{m}, h, \mathcal{H}_{i}, \hat{\mathcal{H}}_{k}\right\}\right)
$$

where $\frac{1}{M} \sum_{m=1}^{M} \operatorname{Pr}\left\{e \mid s_{m}, h, \mathcal{H}_{i}, \hat{\mathcal{H}}_{k}\right\}=2\left(1-\frac{1}{M}\right) Q\left(\sqrt{\frac{d_{m i n, k}^{2}|h|^{2}}{4 \sigma_{\bar{n}_{r}}^{2}}}\right)$

Above, $\operatorname{Pr}\left\{e \mid s_{m}, h, \mathcal{H}_{i}, \hat{\mathcal{H}}_{k}\right\}$ denotes the conditional error probability given the transmitted signal $s_{m}$, channel fading $|h|$, true state of the channel, $\mathcal{H}_{i}$, and sensing decision, $\hat{\mathcal{H}}_{k}$. Also, $Q(a)=\frac{1}{\sqrt{2 \pi}} \int_{a}^{\infty} e^{\frac{-x^{2}}{2}} d x$. Averaging $P_{e, h}$ given in (15) over the fading distribution, we obtain

$$
\begin{aligned}
P_{e} & =\sum_{k=0}^{1} 2 \operatorname{Pr}\left\{\hat{\mathcal{H}}_{k}\right\}\left(\operatorname{Pr}\left\{\mathcal{H}_{0} \mid \hat{\mathcal{H}}_{k}\right\}\left(1-\frac{1}{M}\right) \mathbb{E}_{|h|^{2}}\left[Q\left(\sqrt{\frac{d_{\text {min }, k}^{2}|h|^{2}}{4 \sigma_{\bar{n}_{r}}^{2}}}\right)\right]\right. \\
& \left.+\operatorname{Pr}\left\{\mathcal{H}_{1} \mid \hat{\mathcal{H}}_{k}\right\}\left(1-\frac{1}{M}\right) \mathbb{E}_{|h|^{2}}\left[Q\left(\sqrt{\frac{d_{\text {min }, k}^{2}|h|^{2}}{4\left(\sigma_{\bar{n}_{r}}^{2}+\sigma_{\bar{w}_{r}}^{2}\right)}}\right)\right]\right)
\end{aligned}
$$

We observe above that while the optimal decision rule does not depend on the sensing decisions, the error rates are functions of detection and false alarm probabilities. In the special case of a Rayleigh fading model for which the fading power has an exponential distribution with unit mean, i.e., $f_{|h|^{2}}\left(|h|^{2}\right)=$ $\mathrm{e}^{-|h|^{2}}$, the expectations in the error probability expression can be obtained in closed-form and we can write

$$
\begin{aligned}
& P_{e}=\sum_{k=0}^{1} \operatorname{Pr}\left\{\hat{\mathcal{H}}_{k}\right\}\left[\operatorname{Pr}\left\{\mathcal{H}_{0} \mid \hat{\mathcal{H}}_{k}\right\}\left(1-\frac{1}{M}\right)\left(1-\sqrt{\frac{d_{m i n}, k}{d_{m i n, k}^{2}+8 \sigma_{\bar{n}_{r}}^{2}}}\right)\right. \\
& \left.+\operatorname{Pr}\left\{\mathcal{H}_{1} \mid \hat{\mathcal{H}}_{k}\right\}\left(1-\frac{1}{M}\right)\left(1-\sqrt{\frac{d_{m i n, k}^{2}}{d_{m i n, k}^{2}+8\left(\sigma_{\bar{n}_{r}}^{2}+\sigma_{\bar{w}_{r}}^{2}\right)}}\right)\right] .
\end{aligned}
$$

\section{2) Rectangular QAM transmission under channel sensing} error:

Remark 2: Since $M_{I} \times M_{Q}$ rectangular QAM modulation can be regarded as two independent PAM modulations on the real and imaginary components, the optimal decision rule for $M_{I} \times M_{Q}$ rectangular QAM signaling for any channel sensing decision consists of comparing the real and imaginary components of $y$ with the midpoint between the received signals.

For $M_{I} \times M_{Q}$ rectangular QAM, the minimum distance between $d_{\min , k}$ is given by

$$
d_{\text {min }, k}=\sqrt{\frac{12}{I^{2}+J^{2}-2} \mathcal{E}_{k}} \quad k \in\{0,1\}
$$

where $I, J$ are the modulation size on the in-phase and quadrature components, respectively. The average conditional error probability for $M_{I} \times M_{Q}$ rectangular QAM given an idle channel and corresponding sensing decision can be expressed as

$\operatorname{Pr}\left\{e \mid \mathcal{H}_{0}, \hat{\mathcal{H}}_{k}\right\}=1-\mathbb{E}_{|h|^{2}}\left[\left(1-P_{e, I-P A M}\right)\left(1-P_{e, Q-P A M}\right)\right]$

where $P_{e, I-P A M}$ and $P_{e, Q-P A M}$ denote the average conditional error probabilities of $M_{I}$-PAM and $M_{Q}$-PAM modulations, respectively. These conditional probabilities can easily be found from (16) by using the above $d_{m i n, k}$ expression and replacing $M$ with $M_{I}$ or $M_{Q}$. In (22), we express the error probability averaged over the fading power $|h|^{2}$. In this expression, $\alpha_{0, k}=\sqrt{1+\frac{2\left(I^{2}+J^{2}-2\right) \sigma_{\bar{n}_{r}}^{2}}{3 \mathcal{E}_{k}}} \cdot \operatorname{Pr}\left\{e \mid h, \mathcal{H}_{1}, \hat{\mathcal{H}}_{k}\right\}$ can be derived by following the same steps with $\alpha_{1, k}=$ 


$$
\begin{aligned}
\operatorname{Pr}\left\{e \mid \mathcal{H}_{0}, \hat{\mathcal{H}}_{k}\right\} & =2\left(2-\frac{1}{M_{I}}-\frac{1}{M_{Q}}\right) \mathbb{E}_{|h|^{2}}\left[Q\left(\sqrt{\frac{d_{m i n, k}^{2}|h|^{2}}{4 \sigma_{\bar{n}_{r}}^{2}}}\right)\right]-4\left(1-\frac{1}{M_{I}}\right)\left(1-\frac{1}{M_{Q}}\right) \mathbb{E}_{|h|^{2}}\left[Q^{2}\left(\sqrt{\frac{d_{m i n, k}^{2}|h|^{2}}{4 \sigma_{\bar{n}_{r}}^{2}}}\right)\right] \\
& =\left(2-\frac{1}{M_{I}}-\frac{1}{M_{Q}}\right)\left(1-\frac{1}{\alpha_{0, k}}\right)-2\left(1-\frac{1}{M_{I}}\right)\left(1-\frac{1}{M_{Q}}\right)\left(\frac{2}{\pi} \frac{1}{\alpha_{0, k}} \tan ^{-1}\left(\frac{1}{\alpha_{0, k}}\right)-\frac{1}{\alpha_{0, k}}+\frac{1}{2}\right)
\end{aligned}
$$

$$
P_{e}=\sum_{k=0}^{1} \sum_{i=0}^{1} \operatorname{Pr}\left\{\hat{\mathcal{H}}_{k}\right\}\left\{\operatorname{Pr}\left\{\mathcal{H}_{i} \mid \hat{\mathcal{H}}_{k}\right\}\left[\left(2-\frac{1}{M_{I}}-\frac{1}{M_{Q}}\right)\left(1-\frac{1}{\alpha_{i, k}}\right)-2\left(1-\frac{1}{M_{I}}\right)\left(1-\frac{1}{M_{Q}}\right)\left(\frac{2}{\pi} \frac{1}{\alpha_{i, k}} \tan ^{-1}\left(\frac{1}{\alpha_{i, k}}\right)-\frac{1}{\alpha_{i, k}}+\frac{1}{2}\right)\right]\right\}
$$

$\sqrt{1+\frac{2\left(I^{2}+J^{2}-2\right)\left(\sigma_{\bar{n}_{r}}^{2}+\sigma_{\bar{w}_{r}}^{2}\right)}{3 \mathcal{E}_{k}}}$. Overall, the average symbol error probability for $M_{I} \times M_{Q}$ rectangular QAM can be written as in (23) at the top of the page. The derivation of the expectation of squared Gaussian $Q$ function over Rayleigh fading is given in [10]. Note that we can easily obtain the average symbol error probability for square $M-\mathrm{QAM}$ by setting $M_{I}=M_{Q}=\sqrt{M}$.

\section{NumericAl Results}

In this section, we present numerical results to illustrate the error performance of rectangular QAM modulation schemes under channel sensing errors. Theoretical results are validated through Monte Carlo simulations. Unless mentioned explicitly, the following parameters are employed in numerical computations. It is assumed that noise variance $\sigma_{n}^{2}=0.04$, interference variance $\sigma_{w}^{2}=0.1$ and the power of channel fading coefficient has unit mean. Also, $\operatorname{Pr}\left\{\mathcal{H}_{1}\right\}=0.4$ and $\operatorname{Pr}\left\{\mathcal{H}_{0}\right\}=0.6$. The cognitive user sets the average transmission energy $\mathcal{E}_{1}$ to 1 in the presence of active primary users whereas $\mathcal{E}_{0}=10$ if there is no primary user activity in the channel.

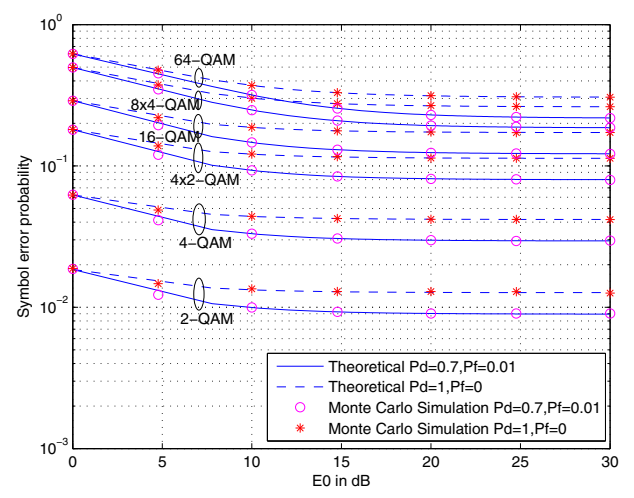

Fig. 1. Average probability of error of $M-\mathrm{QAM}(M=\{2,4,8,16,32,64\})$ signaling vs. average energy $\mathcal{E}_{0}$ in $\mathrm{dB}, \mathcal{E}_{1}=1$.

In Fig. 1, we plot average symbol error probability $P_{e}$ as a function of $\mathcal{E}_{0}$, which is the average transmission energy when the channel is detected as idle. In order to limit the amount of interference on the primary users, the average energy when the channel is detected as busy is fixed at $\mathcal{E}_{1}=1$. As expected, the average probability of error $P_{e}$ decreases with increasing $\mathcal{E}_{0}$ and a higher modulation size leads to higher error rates at the same $\mathcal{E}_{0}$ level over the Rayleigh fading channel in the presence of Gaussian mixture noise. We also observe that as $\mathcal{E}_{0}$ increases,
$P_{e}$ curves in all cases approach some error floor. This is due to the assumption that $\mathcal{E}_{1}$ is fixed at 1 . Therefore, at large values of $\mathcal{E}_{0}$, the average error probability is dominated by the frequency of errors occurring during transmissions when the channel is detected as busy and transmission power is lowered to $\mathcal{E}_{1}=$ $1=0 \mathrm{~dB}$. In order to avoid such error floors, cognitive users may opt to stop transmission when the channel is sensed as busy, which comes at the cost of lower data transmission rates.

Another interesting observation is the following. In Fig. 1, $P_{e}$ is plotted for two different pairs of detection and false alarm probabilities, denoted by $P_{d}$ and $P_{f}$, respectively. In the first scenario, channel sensing is perfect; hence, $P_{d}=1$ and $P_{f}=0$. In the second scenario, channel sensing is performed with $P_{d}=0.7$ and $P_{f}=0.01$. We notice that in the first scenario we have higher $P_{e}$ compared with that in the second scenario. This is due to the fact that in imperfect channel sensing, miss-detections occur and cognitive users at these times transmit at higher energy levels even though primary users are active. In such cases, lower error probabilities can be attained. Hence, imperfect sensing tend to benefit the cognitive users while leading to increased interference on the primary users. In the figure, we also observe that as $\mathcal{E}_{0}$ increases, the gap between the error rate performances under perfect and imperfect channel sensing initially increases and then remains constant.

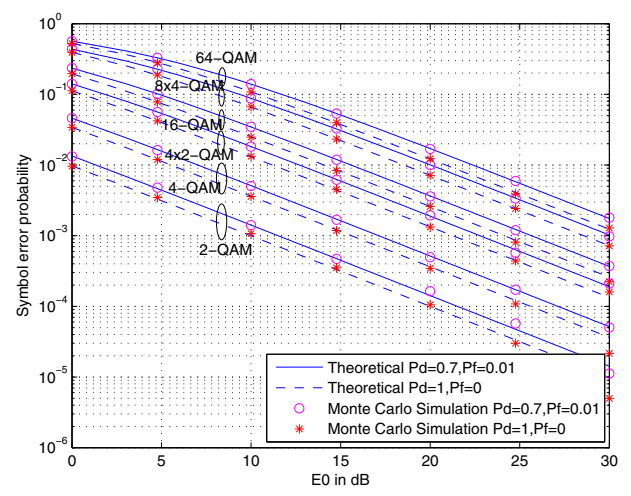

Fig. 2. Average probability of error of $M-\mathrm{QAM}(M=\{2,4,8,16,32,64\})$ signaling vs. average energy $\mathcal{E}_{0}$ in $\mathrm{dB}$.

In Fig. 2, we plot average symbol error probability $P_{e}$ as a function of $\mathcal{E}_{0}$ when the cognitive users transmit data only when the channel is sensed to be not occupied by the primary users (i.e., $\mathcal{E}_{1}=0$ ). Increasing $\mathcal{E}_{0}$ leads to decreasing $P_{e}$ without an 
error floor, as expected. It is also observed that perfect channel sensing improves the error rate performance of cognitive users, which is in contrast with the scenario observed in Fig. 1. Note that if sensing is perfect, cognitive users transmit only if the channel is idle and experience only the background noise $n$. On the other hand, under imperfect sensing, cognitive users transmit in miss-detection scenarios as well, in which they are affected by both the background noise and primary user interference $w$, leading to higher error rates.

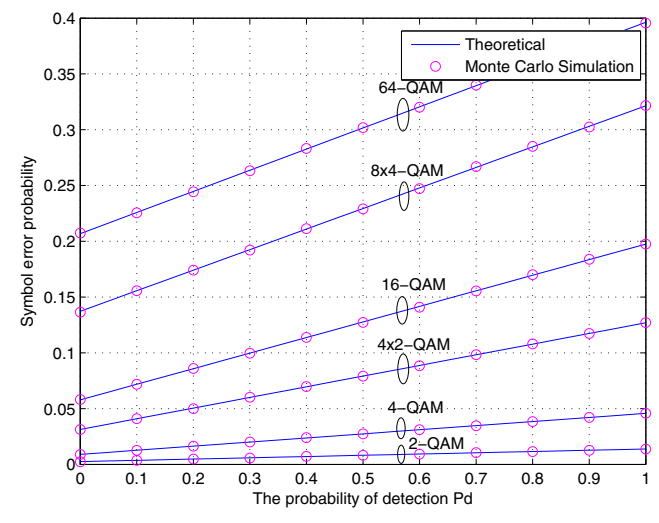

Fig. 3. Average symbol error probability of $M-\mathrm{QAM}(M=\{2,4,8,16,32$, 64\}) signaling vs. detection probability $P_{d} . \mathcal{E}_{0}=10$ and $\mathcal{E}_{1}=1$

In Fig. 3, we display the average symbol error probability as a function of the detection probability $P_{d}$, where $P_{f}$ is set to 0.1 . Here, we also assume that $\mathcal{E}_{0}=10$ and $\mathcal{E}_{1}=1$. Hence, transmission with lower power occurs in busy-detected channels. It is seen that error rate performances of $M-\mathrm{QAM}$ signaling worsen as detection probability increases because of the same reasoning as before. More reliable detection of primary user activity leads to more frequent low-energy transmissions than would otherwise be. Or equivalently, at lower detection probabilities, more miss-detections occur and the cognitive users transmit at high energy levels more often. Note that the gain in the error performance in such cases is realized at the expense of higher levels of interference on the primary users. Therefore, in order to protect the primary users, lower bounds on $P_{d}$ should be imposed. One additional remark from the figure is that as $M$ increases, the symbol error probability increases more rapidly.

In Fig. 4, we analyze the average probability of error $P_{e}$ as a function of the false alarm probability $P_{f}$, where $P_{d}=0.7$. It is observed that $P_{e}$ increases with increasing false alarm probability. Hence, degradation in the sensing performance in terms of increased false alarm probabilities leads to degradation in the error performance. As false-alarms become more frequent, cognitive users sense the channel busy more often even if the channel is not occupied by the primary users. In those cases, lower transmission energy is used to limit the interference on the primary users and lower error performance is achieved.

\section{CONCLUSion}

We have studied the error probability of cognitive radio transmissions with imperfect channel sensing. We have assumed that following channel sensing, the cognitive transmitter sends

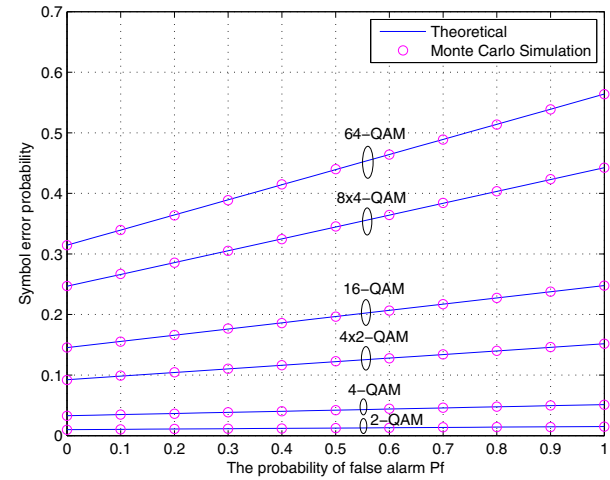

Fig. 4. Average symbol error probability of $M-$ QAM $(M=\{2,4,8,16,32$, 64\}) signaling vs. false alarm probability $P_{f} \cdot \mathcal{E}_{0}=10$ and $\mathcal{E}_{1}=1$.

equiprobable rectangular QAM signals over a flat-fading channel. In the presence of sensing errors, the secondary receiver is shown to experience Gaussian mixture noise. Under these assumptions, we have proved that midpoints between the signals are optimal thresholds for the detection of $M-\mathrm{PAM}$ and rectangular QAM signals under any sensing decision. Symbol error probabilities are shown to be in general dependent on sensing performance through the detection and false alarm probabilities. For instance, we have observed that as the detection probability increases, error probabilities increase as well due to transmissions with lower energy in the presence of detected primary user activity. Because of the same reason, error probability is also shown to increase with increasing false-alarm probability. Additionally, we have noted that if the cognitive users transmit only when the channel is sensed as idle, improved sensing performance leads to lower error rates.

\section{REFERENCES}

[1] A. Ghasemi and E. S. Sousa, "Spectrum sensing in cognitive radio networks: Requirements, challenges and design trade-offs," IEEE Comm. Mag., vol. 46, no, 4, pp. 32-39, Apr. 2008.

[2] E. Hossain, D. Niyato, and Z. Han, Dynamic Spectrum Access and Management in Cognitive Radio Networks, Cambridge University Press, 2009.

[3] E. Axell, G. Leus, E. G. Larsson, and H. V. Poor, "Spectrum sensing for cognitive radio: State-of-the-art and recent advances," IEEE Signal Process. Mag., vol. 29, no. 3, pp. 101-116, May 2012.

[4] A. Ghasemi and E. S. Sousa, "Fundamental limits of spectrum-sharing in fading environments," IEEE Trans. Wireless Commun., vol. 6, no. 2, pp. 649-658, Feb. 2007.

[5] Y.-C. Liang, Y. Zeng, E. C. Y. Peh, A. T. Hoang, "Sensing-throughput tradeoff for cognitive radio networks," IEEE Trans. Wireless Commun., vol. 7, no. 4, pp. 1326-1337, Apr. 2008.

[6] H.A. Suraweera, J. P. Smith, and M. Shafi, "Capacity limits and performance analysis of cognitive radio with imperfect channel knowledge," IEEE Trans. Veh. Technol., vol. 59, no. 4, pp. 1811-1822, May 2010.

[7] D. Xu, Z. Feng, and P. Zhang, "Minimum average BER power allocation for fading channels in cognitive radio networks," in Proc. IEEE WCNC, 2011, pp. 78-83, Mar. 2011.

[8] L. Li, P. I. Derwin, and M. Pesavento , "Symbol error rate analysis in multiuser underlay cognitive radio systems," in Proc. IEEE PIMRC, 2011, pp. 681-684, Sept. 2011.

[9] H. V. Poor, An Introduction to Signal Detection and Estimation. New York: Springer-Verlag, 1994.

[10] M. K. Simon and M. S. Alouini, "A unified approach to the performance analysis of digital communication over generalized fading channels," in Proc. IEEE, vol. 86, no. 9, pp. 1860-1877, Sep. 1998. 